\title{
Study on Diamagnetic Levitation Structure in Electromagnetic Vibration Energy Harvester
}

\author{
Zhenyu Zhang, ${ }^{1}$ Kun Zhang, ${ }^{1}$ Zhiyong Duan, ${ }^{2}$ and Yufeng Su ${ }^{1 *}$ \\ ${ }^{1}$ School of Mechanical Engineering, Zhengzhou University, Zhengzhou 450001, China \\ ${ }^{2}$ School of Physical Engineering, Zhengzhou University, Zhengzhou 450001, China
}

(Received December 1, 2017; accepted Janualy 9, 2018)

Keywords: diamagnetic levitation, vibration energy harvester, simulation, nonlinear vibration, bistability

In this paper, we proposed an electromagnetic vibration energy harvester with a diamagnetic levitation structure, which consists of a lifting magnet, two highly oriented pyrolytic graphite (HOPG) sheets, and a floating magnet. The dynamic characteristics of the diamagnetic levitation structure are critical to the performance of the energy harvester. The diamagnetic and magnetic forces were calculated in $\mathrm{COMSOL}^{\mathrm{TM}}$, and the bistable phenomenon of the diamagnetic levitation structure was found and verified experimentally. The nonlinear characteristics of the diamagnetic levitation structure were studied in Simulink. Under different external excitations, the intrawell and interwell vibrations were observed in phase diagrams. When the excitation frequency is $1 \mathrm{~Hz}$ and the amplitude is $8.1 \mathrm{~mm}$, the low-energy region is similar to the high-energy region, which is beneficial to harvest energy.

\section{Introduction}

Nowadays, wireless sensor nodes, medical implantable devices, and wireless transmitters are widely used. ${ }^{(1)}$ However, the key technical problem that must be solved is how to adopt easily applicable methods to supply power for these devices. ${ }^{(2)}$ They rely mainly on batteries which must be replaced when they run out of power. In many cases, it is not easy to replace the used batteries or the cost of replacing the battery is very high, such as the batteries in heart pacemakers and sensors embedded in bridges. Therefore, it is necessary to supply sustainable power to these miniature electronic devices, in order to achieve their autonomous and selfsustained long-term operation. ${ }^{(3)}$ Ambient vibration sources are prevalent in many everyday applications, making them suitable power sources for low-power electronic devices. ${ }^{(4)}$ The vibration energy harvester is one of the effective replacements for traditional chemical batteries. It harvests energy from ambient energy sources and supplies power to the electronic devices.

Many microelectricity supply schemes have been proposed by scholars throughout the world, such as piezoelectric, electromagnetic, photoelectric, thermoelectric, and magnetostrictive energy harvesters. ${ }^{(5-10)}$ Heat, light, wind, and mechanical vibration have their respective advantages and disadvantages. Mechanical vibration is widespread, and the vibration energy

*Corresponding author: e-mail: yufengsu@zzu.edu.cn http://dx.doi.org/10.18494/SAM.2018.1835 
harvester has become an active topic. Mechanical vibrations have various forms in the ambient environment, such as body motion, heartbeats, vehicle movement, engine vibration, and bridge vibration. Vibration energy harvesters reported in the literature are typically based on the linear system, ${ }^{(11)}$ which leads to lower output power when the natural frequency does not match the excitation frequency. A cantilever beam ${ }^{(12)}$ and a mechanical spring ${ }^{(13)}$ are often used in the linear vibration energy harvester. Ansari and Anin ${ }^{(14)}$ introduced an energy harvester with a folded fan for energy harvesting from heartbeat vibrations. However, the device is relatively insensitive to low-frequency vibration owing to the linear system. The narrow response bandwidth of the linear vibration energy harvesters limits the application fields. ${ }^{(15)}$ To obtain an energy harvester that can be used in many fields, a nonlinear structure is introduced into the energy harvester. Karami and Inman ${ }^{(16)}$ and Zhou et al. ${ }^{(17)}$ studied the nonlinearity of piezoelectric-electromagnetic hybrid energy harvesters. Yildirim et al. ${ }^{(18)}$ designed an energy harvester with a clamped-clamped beam and a magnet. The frequency band of the energy harvester has been broadened significantly. Abed et al. ${ }^{(19)}$ proposed a multimodal vibration energy harvesting approach based on arrays of coupled levitated magnets. A normalized power of $20.2 \mathrm{~mW} \cdot \mathrm{cm}^{-3} \cdot \mathrm{g}^{-2}$ was obtained under the operating frequency range of 4.6-14.5 $\mathrm{Hz}$ with a bandwidth of $190 \%$.

The bandwidth of the vibration system can be effectively broadened by nonlinear vibration systems that have monostable, ${ }^{(20)}$ bistable, $^{(21-23)}$ and multistable ${ }^{(24)}$ states. Magnetic force is often used to build nonlinear vibration energy harvesters. Andò et al. ${ }^{(25)}$ proposed a 2D wideband bistable vibration energy harvester with a small magnet added to the tip of the cantilever. The bandwidth was widened effectively and the maximum output power was $5.6 \mu \mathrm{W}$ with a resistive load of $330 \mathrm{k} \Omega$. Podder and Roy ${ }^{(26)}$ discussed a wideband, bistable vibrational micropower generator using a folded cantilever beam. The half-power bandwidth of the nonlinear bistable system was increased by $5 \mathrm{~Hz}$ with respect to the linear counterpart.

In this paper, an electromagnetic vibration energy harvester with a bistable structure is proposed. In Sect. 2, we describe the structure of the diamagnetic energy harvester. In Sect. 3, we analyze the magnetic and diamagnetic forces exerted on the floating magnet in $\mathrm{COMSOL}^{\mathrm{TM}}$, and the bistable phenomenon of the diamagnetic levitation structure is found and verified experimentally. In Sect. 4, we discuss the nonlinear characters of the diamagnetic levitation structure in the energy harvester. Finally, In Sect. 5, we present the concluding remarks.

\section{Structure of Energy-harvesting Device}

As shown in Fig. 1, the energy harvester is composed of a diamagnetic levitation structure and two induced coils. The diamagnetic levitation structure consists of a lifting magnet (LM), an upper highly oriented pyrolytic graphite (HOPG) sheet, a floating magnet (FM), and a lower HOPG sheet, from top to bottom. In the structure, the floating magnet is levitated between two HOPG sheets. The poles of the lifting and floating magnets are arranged in the same direction. Thus, an attractive force that can overcome a part of the gravity of the floating magnet is generated between the two magnets. The magnetic susceptibility $\left[(-8,-8,-45) \times 10^{-5}\right]$ of the HOPG is less than 0 , which means that the HOPG sheets repel the magnetic fields. Therefore, 


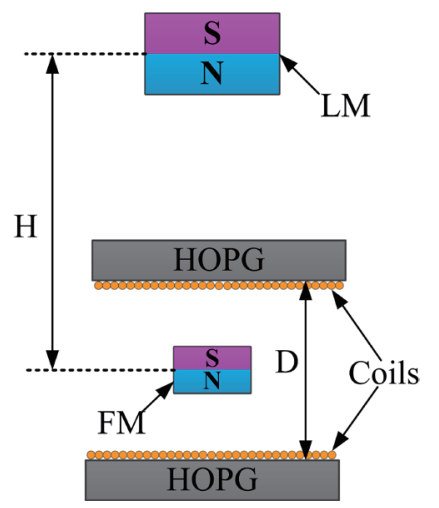

Fig. 1. (Color online) Schematic of vibration energy harvester.

the HOPG sheet is introduced to the energy harvester to provide a repulsive force (diamagnetic force). Under the combined effects of magnetic force, diamagnetic force, and gravity, the floating magnet can achieve a stable levitation. When the energy harvester is excited by an external vibration, a relative movement will be generated between the floating magnet and the induced coils. Then the magnetic flux in the coil will change, and an induction voltage will be obtained. The parameters of the energy harvester are illustrated in Table 1.

\section{Analysis of Diamagnetic Levitation Structure and Experimental Verification}

The calculations of the magnetic force and diamagnetic force are simulated in the finite element analysis (FEA) software COMSOL ${ }^{\mathrm{TM}}$. As shown in Figs. 2(a) and 2(b), the model is encircled by an air domain that is divided into outer and inner layers. The outer layer is infinite with a coarse grid to simulate the dispersion field of the magnet. The inner layer is a finite field with a fine grid to improve the simulation accuracy. The unit type is a free subdivision tetrahedral mesh. In Figs. 2(a) and 2(b), the numbers of model elements are 661370 and 920624 , respectively. In the simulation, we take the symmetrical plane of the two HOPG sheets as the zero plane and the upward direction as positive. The simulation results are shown in Fig. 3.

In Fig. 3, $F_{M G}$ is the resultant force of magnetic force and gravity, and $F_{D}$ is diamagnetic force exerted on the floating magnet. As shown in Fig. 3, the diamagnetic force of the floating magnet $F_{D}$ is origin-symmetric. The nonlinearity of the diamagnetic force becomes stronger with an increase in the spacing $D$. There are three points of intersection between $F_{M G}$ and $F_{D}$. In other words, the diamagnetic levitation structure has three equilibrium points: upper, middle, and bottom equilibrium points from top to bottom. The three equilibrium points will overlap at one point when the spacing $D$ is very small, for example, $D=2 \mathrm{~mm}$. With an increase in the spacing $D$, the upper, middle, and bottom equilibrium points gradually separate. In addition, the equilibrium state of the floating magnet will be altered by adjusting the distance $H$ between the lifting and floating magnets, such as the number of equilibrium points. With an increase in $H$, the middle and bottom equilibrium points gradually move away from the zero plane, and the upper equilibrium points gradually approach the zero plane. 
Table 1 Main parameters of energy harvester.

\begin{tabular}{lcc}
\hline Component & Material & Dimensions (mm) \\
\hline LM & NdFeB (N52) & $\varnothing 4.8 \times 3.2$ \\
HOPG sheet & HOPG & $\varnothing 10 \times 2.5$ \\
Coil & Copper & $\varnothing 0.02$ \\
FM & NdFeB (N42) & $\varnothing 3.2 \times 1.6$ \\
\hline
\end{tabular}

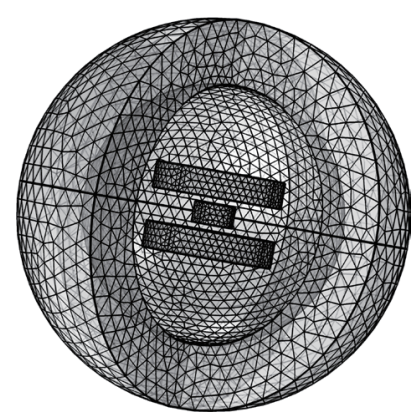

(a)

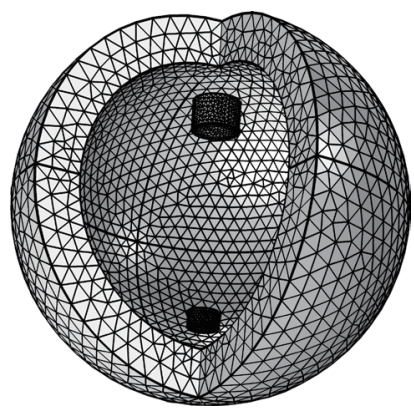

(b)

Fig. 2. FEA simulation model of diamagnetic levitation structure: (a) calculation model of diamagnetic force and (b) calculation model of magnetic force.

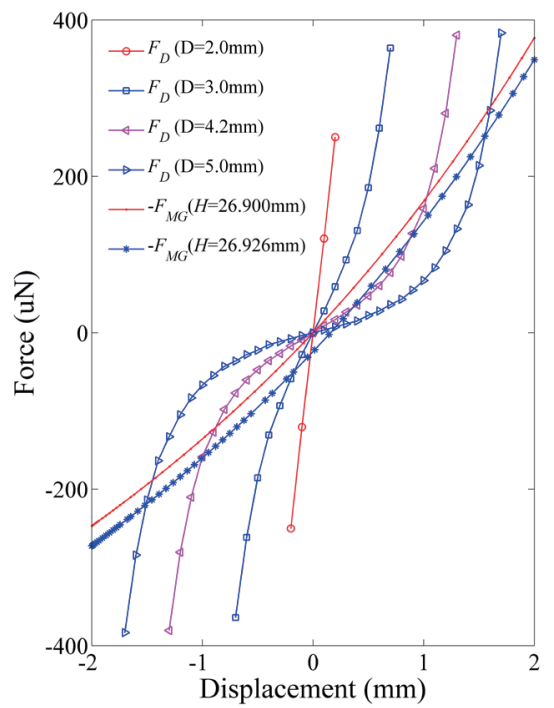

Fig. 3. (Color online) Force curve of the floating magnet.

The equilibrium point of the floating magnet is not necessarily the stable equilibrium point. A stable equilibrium point must have a minimum potential energy. That is, when the floating magnet is levitated stably, the floating magnet is at the bottom of the potential well. It can be seen from Fig. 4(a) that the number and depth of the potential well will change with $D$. When $D=2 \mathrm{~mm}$, the potential curve has only one potential well where the floating magnet can be levitated stably. With an increase in $D$, the middle equilibrium point will turn into an unstable equilibrium point. The upper and bottom equilibrium points are at the bottom of the potential well where the floating magnets can be levitated stably. The phenomenon shows the bistability of diamagnetic levitation. Figure 4(b) shows the equilibrium state of the floating magnet. When the floating magnet is stationary, it must be at the bottom of the well. The floating magnet reciprocates within one potential well when it is excited under a weak external excitation. When the external excitation is sufficiently large, the floating magnet can overcome the potential barrier, and then it can reciprocate between two potential wells. For the energy 


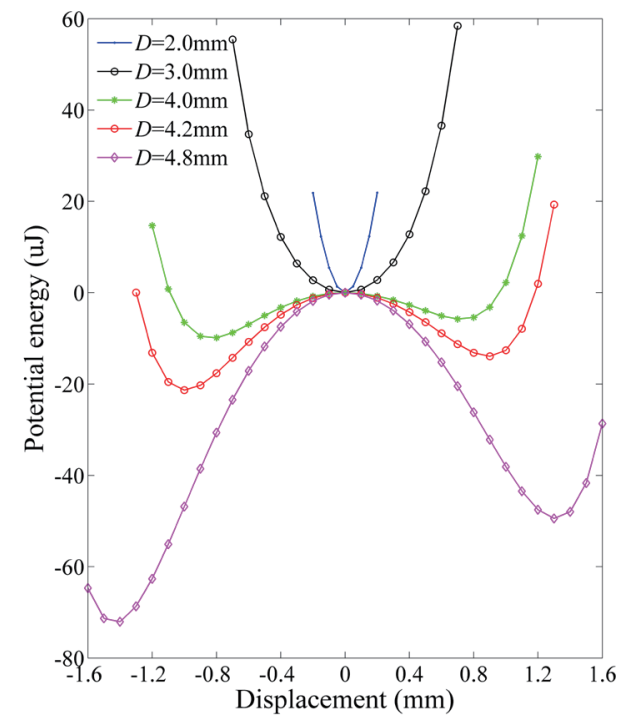

(a)

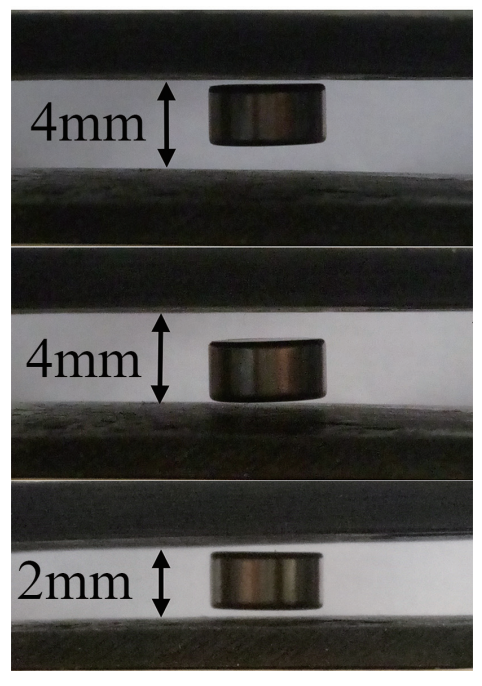

(b)

Fig. 4. (Color online) Bistable state of the diamagnetic levitation structure: (a) potential energy curve of floating and (b) equilibrium states.

harvester, large displacement vibration will lead to a dramatic change in the magnetic field of the induced coils, and it is beneficial for increasing the harvester's output voltage. The depth of potential wells and the potential barrier are the key factors that determine whether the floating magnet can jump between the two potential wells. Jumping from the deep potential well to the shallow one takes more energy than vice versa. Thus the depth of the deep potential well determines whether interwell vibration can occur. With the increase in $D$, the potential wells will become deeper, and the potential difference between the two potential wells will increase.

By adjusting the distance $H$ between the lifting and floating magnets, the resultant force of the floating magnet can be changed, and the potential energy curve will be changed. The effect of $\mathrm{H}$ on the resultant force of the floating magnet was studied when $D=4.2 \mathrm{~mm}$. The change in the potential energy with $H$ is indicated in Fig. 5(a). The depth of the potential well can be adjusted by changing the distance $H$. Figure 5(b) shows the change in the depth of potential wells with $H$. When $H$ increases, the depth in the positive potential well will increase and the negative potential well will decrease. When $H=26.926 \mathrm{~mm}$, the two potential wells have the same depth. Meanwhile, the potential barrier between positive and negative wells is the smallest and interwell vibration can easily occur.

\section{Dynamic Response Analysis of Diamagnetic Levitation Structure}

The vibration system can be equivalent to a spring-mass model, and the equivalent model of the system is shown in Fig. 6. In the model, the floating magnet is subjected to the magnetic force $F_{M}$, gravity $F_{G}$, diamagnetic force $F_{D}$, damping force $F_{K}$, and external excitation $F_{e}$. The vibration equation can be expressed as

$$
m a^{2}-F_{M G}+F_{D}+F_{K}=F_{e}
$$




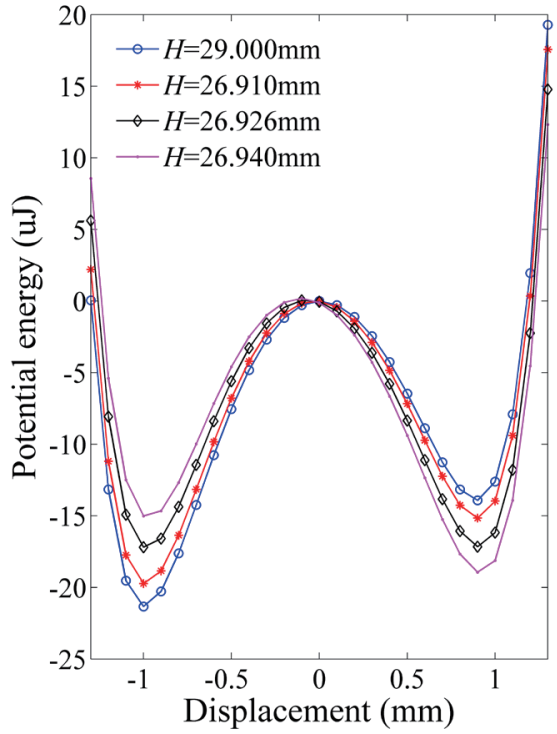

(a)

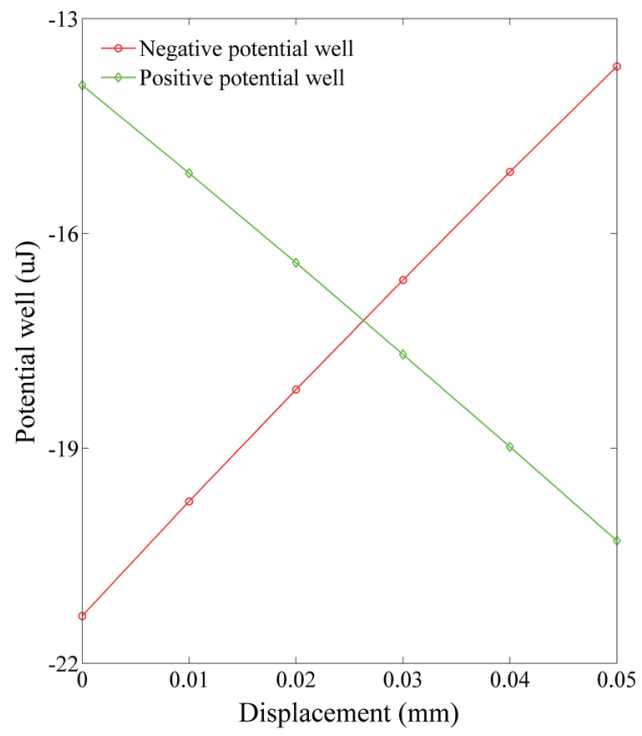

(b)

Fig. 5. (Color online) (a) Potential energy with $D=4.2 \mathrm{~mm}$ and (b) depth of potential well with $D=4.2 \mathrm{~mm}$.

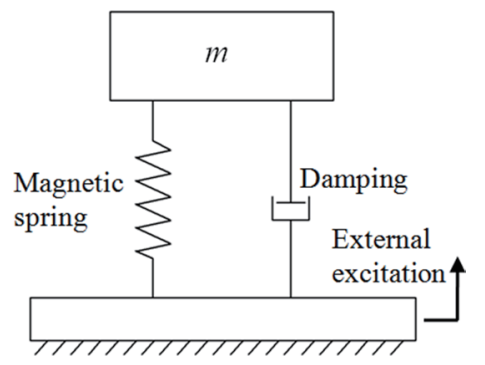

Fig. 6. Equivalent spring-mass model.

where $m$ is the mass of the floating magnet, $a$ is the acceleration, $F_{M G}=F_{M}-F_{G}, F_{K}$ is the damping, and $F_{e}$ is the external excitation in the environment.

Owing to the nonlinearity of the diamagnetic levitation structure, the floating magnet has complicated response characteristics to external excitation. The amplitude-frequency response curve of the diamagnetic levitation structure is shown in Fig. 7. The amplitude response curve of the floating magnet is turned to the right, which is the classic characteristic of the hardening spring. With the increase in the excitation amplitude, the phenomenon becomes more obvious. The diamagnetic force of the floating magnet shows high nonlinearity, which is the reason for the right tilt phenomenon of the frequency response curve. Moreover, as the excitation amplitude increases, the right tilt phenomenon becomes increasingly intense. When the external excitation amplitude is very small, the amplitude response curve of the floating magnet is similar to linear.

To understand the response of the diamagnetic levitation structure to the external excitation, a simulation model was built in Simulink, as shown in Fig. 8. In the simulation model, the distance between the two HOPG sheets is $4.2 \mathrm{~mm}$ and the excitation is a sine wave generated 


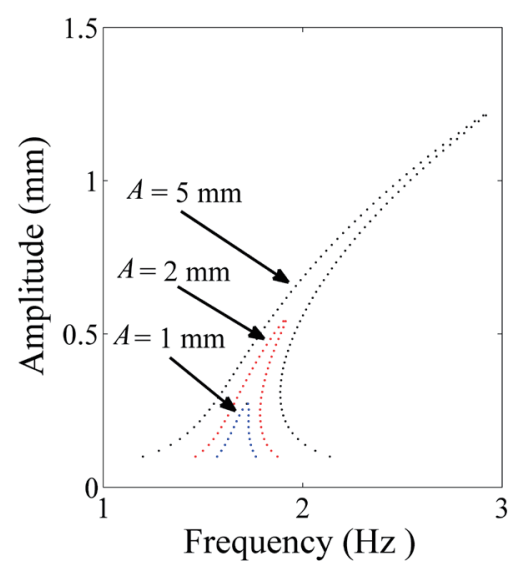

Fig. 7. (Color online) Amplitude-frequency response of the diamagnetic levitation structure.

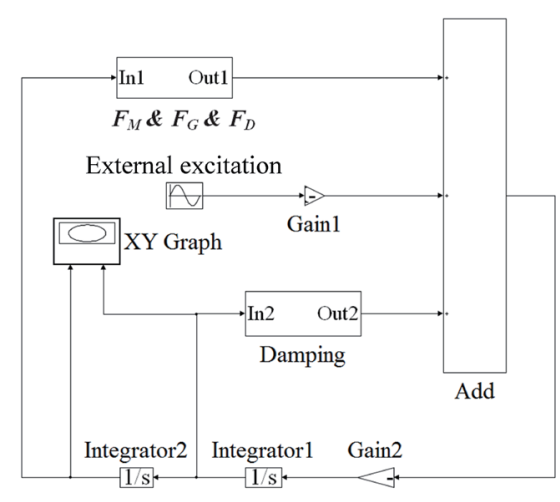

Fig. 8. Simulink model for the diamagnetic levitation structure.

by a signal generator module. The excitation signal was passed through the Simulink wiring diagram, which calculated the phase diagram for the diamagnetic levitation structure.

When the diamagnetic levitation structure is asymmetrical, the phase diagram of free vibration of the floating magnet was obtained and is shown in Fig. 9(a). In the simulation, the initial position of the floating magnet is set at the negative limit point. It can be seen from Fig. 9(a) that the floating magnet can overcome the barrier between the two potential wells. Then, the diamagnetic levitation structure was adjusted to a symmetrical form, and the remaining conditions remain unchanged. The phase diagram of free vibration of the floating magnet in the symmetrical structure is illustrated in Fig. 9(b). The floating magnet cannot overcome the barrier between the two potential wells in the symmetric structure. The floating magnet can only reciprocate vibration in the negative well and could not enter the other potential well. Comparing the two simulation results, we find that asymmetric structures can easily realize interwell vibration. The larger the amplitude of the floating magnet, the greater the magnetic flux in the coil; this phenomenon is beneficial to the output of the energy harvester.

The magnitude of the output voltage is determined by the velocity of the floating permanent magnet. In the same position, the higher the speed of motion of the floating magnet, the higher the output voltage of the energy harvester. The relationship between velocity and displacement is clearly shown in the phase diagram. The vibration of the floating permanent magnet includes the start-up state and the steady state. The phase diagrams for the vibration of the floating permanent magnet under different external excitations are shown in Fig. 10. The phase diagram of the start-up state is very complicated, which is not helpful in observing the vibration of the floating magnet under the steady state, so only the phase diagram of the steady state is shown in Fig. 10. The excitation amplitudes are 4, 7.5, 8.1, and $9.6 \mathrm{~mm}$, and the frequency is $1 \mathrm{~Hz}$. When the external excitation amplitude is $4 \mathrm{~mm}$, the floating magnet cannot cross the interwell barrier, so the floating magnet can only reciprocate in a potential well, as shown in Fig. 10(a). As the external excitation amplitude increases, the trajectory of the floating magnet extends to the other potential well, as shown in Fig. 10(b). Under the excitation amplitude of $8.1 \mathrm{~mm}$, the phase diagram of the floating magnet is as shown in Fig. 10(c). The low-velocity orbit of the 


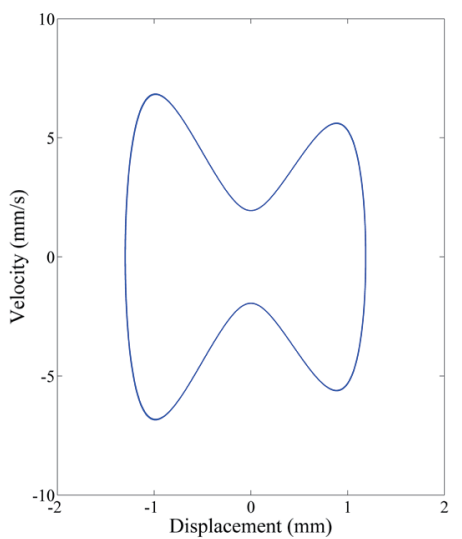

(a)

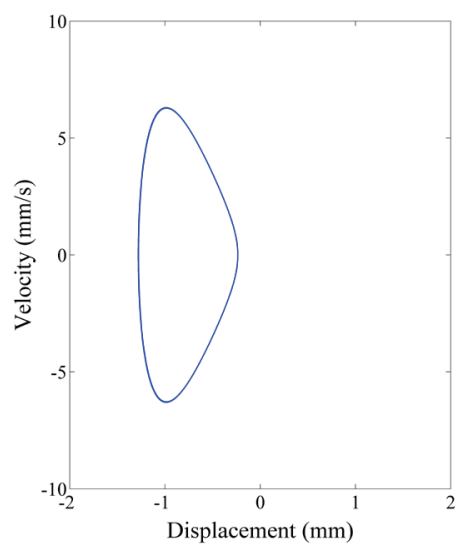

(b)

Fig. 9. (Color online) Phase diagram of free vibration: (a) asymmetrical and (b) symmetrical structures.

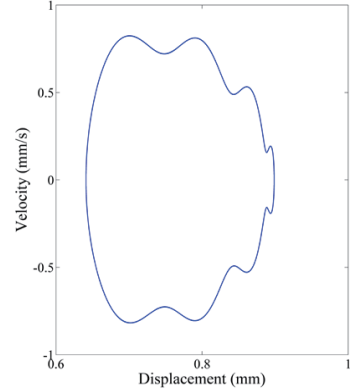

(a)

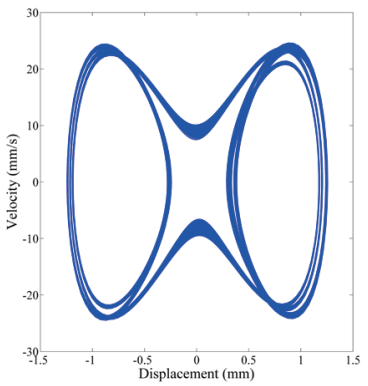

(c)

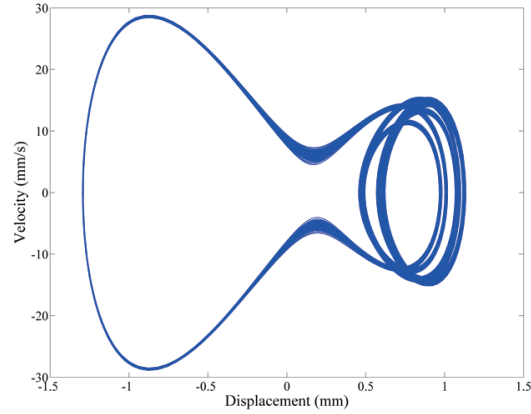

(b)

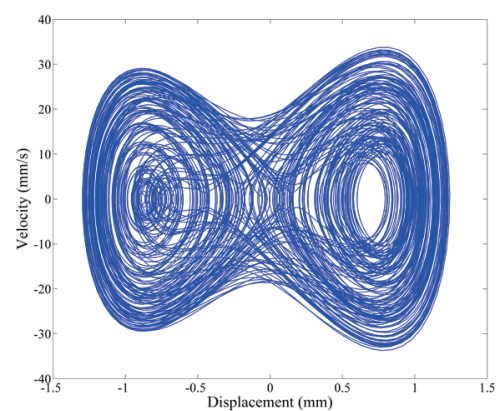

(d)

Fig. 10. (Color online) Phase diagram of the diamagnetic levitation structure under the excitation frequency of 1 $\mathrm{Hz}$ and different amplitudes of (a) 4, (b) 7.5, (c) 8.1 , and (d) $8.1 \mathrm{~mm}$.

floating magnet is extended, and the velocity of the floating magnet is increased. When the amplitude of the external excitation increases to $9.6 \mathrm{~mm}$, the trajectory of the floating magnet becomes cluttered and unpredictable, as shown in Fig. 10(d). This phenomenon shows that the motion of the floating magnet is chaotic, which is not beneficial to harvesting vibration energy. 
A planar spiral coil is selected as the induction coil with $0.4 \mathrm{~mm}$ inner radius and $3.6 \mathrm{~mm}$ outer radius. When the external excitation amplitude is $8.1 \mathrm{~mm}$ with the frequency of $1 \mathrm{~Hz}$, a voltage of about $5 \mathrm{mV}$ is simulated in each one-layer coil. In the future, optimization of the coils and the floating magnet will be studied to improve the performance of the harvester.

\section{Conclusions}

In this paper, the forces exerted on the floating magnet were calculated by $\mathrm{COMSOL}^{\mathrm{TM}}$. The bistable phenomenon of the diamagnetic levitation structure was found through simulation, and was verified experimentally by adjusting the distance between the two HOPG sheets. The characteristics of the symmetric and asymmetric bistable structures were studied by analyzing the potential well of the floating magnet. It was found that the asymmetric bistable structure is more advantageous. A Simulink model was built to study the nonlinearity of the diamagnetic structure with an asymmetrical bistable feature. Under different external excitations, the response characteristics of the floating magnet were studied. High- and low-energy regions were found in the motion trajectories of the floating magnet. The low-energy region expanded as the amplitude of the external excitation increased. Under the excitation frequency of $1 \mathrm{~Hz}$ and the amplitude of $8.1 \mathrm{~mm}$, the low-energy region was similar to the high-energy region, which is beneficial to harvesting energy. When the excitation amplitude was $9.6 \mathrm{~mm}$, the motion of the floating magnet became chaotic. Finally, a voltage about $5 \mathrm{mV}$ was simulated in the one-layer coil. The energy harvester easily responded to vibration frequencies lower than 10 Hz. On the basis of the analytical and experimental results presented in this work, the device will be optimized and the prototype of the energy harvester will be fabricated to further study its work performance.

\section{Acknowledgments}

This work is supported by the National Natural Science Foundation of China under Grant No. 51475436, the Key Scientific and Technological Project of Henan Province under Grant No. 152102210042 and the 2018 Group of Science and Technology Innovation in Universities of Henan Province under Grant No. 18IRTSTHN016.

\section{References}

1 M. Balato, L. Costanzo, and M. Vitelli: Int. J. Electr. Power. 84 (2017) 111.

2 R. W. Chen, L. Ren, H. K. Xia, X. W. Yuan, and X. J. Liu: Sens. Actuators, A. 230 (2015) 1.

3 H. Zhang, L. R. Corr, and T. W. Ma: Mech. Syst. Signal Process. 101 (2018) 55.

4 T. Yildirim, M. H. Ghayesh, W. H. Li, and G. Alici: Renewable Sustable Energy Rev. 71 (2017) 435.

5 Y. Li and R. H. Shi: EURASIP J. Wireless Comman. 1 (2015) 179.

6 P. Markowski: Microelectron. Int. 31 (2014) 176.

7 N. B. Bu, Y. A. Huang, Y. J. Ding, and Z. P. Yin: Sens. Mater. 28 (2016) 757.

8 F. Khan, F. Sassani, and B. Stoeber: Microsyst. Technol. 20 (2014) 1323.

9 A. Matus-Vargas, H. G. Gonzalez-Hernandez, B. S. Chan, A. Palacios, P. L. Buono, V. In, S. Naik, A. Phipps, and P. Longhini: Int. J. Bifurcation Chaos 25 (2015) 1550026.

10 R. Hamid and M. R. Yuce: Sens. Actuators, A 257 (2017) 198. 
B. Andò, S. Baglio, A. R. Bulsara, and V. Marletta: Sens. Actuators, A 211 (2014) 153.

H. Yu, J. L. Zhou, X. Z. Yi, H. Wu, and W. Wang: Microelectron. Eng. 131 (2015) 36.

K. Tao, S. W. Lye, J. M. Miao, and X. Hu: Microelectron. Eng. 135 (2015) 32.

4 M. H. Ansari and K. M. Amin: J. Appl. Phys. 119 (2016) 1540.

15 K. V. Selvan and M. S. M. Ali: Renew Sust. Energ. Rev. 54 (2016) 1035.

16 M. A. Karami and D. J. Inman: J. Sound Vib. 33 (2011) 5583.

17 S. X. Zhou, J. Y. Cao, D. J. Inman, J. Lin, and D. Li: J. Sound Vib. 373 (2016) 223.

18 T. Yildirim, M. H. Ghayesh, W. H. Li, and G. Alici: Energy Convers. Manag. 126 (2016) 247.

19 I. Abed, N. Kacem, N. Bouhaddi, and M. L. Bouazizi: VTT Symp. 25 (2016) 025018.

20 A. M. Elshurafa, K. Khirallah, H. H. Tawfik, A. Emira, A. K. S. A. Aziz, and S. M. Sedky: J. Microelectromech. Syst. 20 (2011) 943.

21 H. Vocca, I. Neri, F. Travasso, and L. Gammaitoni: Appl. Energy 97 (2012) 771.

22 Z. Y. Zhou, W. Y. Qin, and P. Zhu: Energy 126 (2017) 527.

23 H. B. Fang and K. W. Wang: J. Sound Vib. 391 (2017) 153.

24 J. S. Fernando and Q. Sun: J. Renew. Sustainable Ener. 7 (2015) 053110.

25 B. Andò, S. Baglio, F. Maiorca, and C. Trigona: Sens. Actuators, A 202 (2013) 176.

26 P. Podder and A. A. S. Roy: Sens. Actuators, A 227 (2015) 39.

\section{About the Authors}

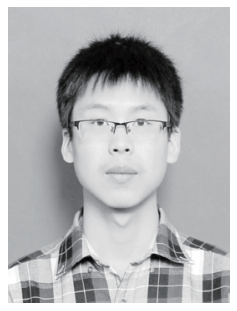

Zhenyu Zhang received his B.S. degree from Shenyang University of Technology, China, in 2015. He is now a master's degree student in the School of Mechanical Engineering, Zhengzhou University. His research interest is on the micro-diamagnetic vibration energy harvester.

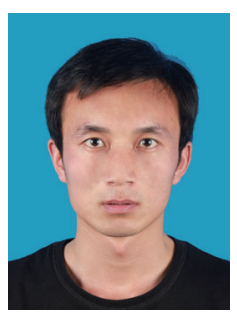

Kun Zhang received his M.S. degree from Zhengzhou University, China, in 2015 and is now a doctor's degree student in the School of Mechanical Engineering, Zhengzhou University. His research interest is on the microdiamagnetic vibration energy harvester.

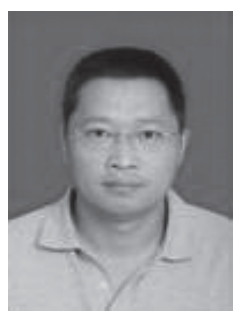

Zhiyong Duan received his Ph.D. degree in microelectronics and solid state electronics at Shanghai Jiao Tong University, China, in 2005. He is an associate professor in the Physical Engineering College, Zhengzhou University. His research interests include piezoelectric actuators, high-precision positioning systems, nanoimprint lithography, interconnects, novel metamaterials texture, and MILC.

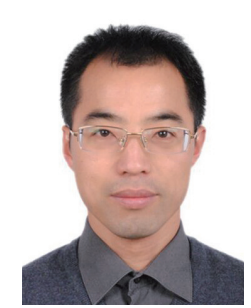

Yufeng Su received his Ph.D. degree from Shanghai Jiao Tong University, China, in 2006. He is a professor in the School of Mechanical Engineering, Zhengzhou University. His research interests include microvibration energy harvesters and microactuators. 